\title{
PODER ECONÓMICO E INFLUENCIA SOCIAL: LOS RETOS DE LA CONCEN'TRACIÓN MEDIÁTICA \\ PARA LA DEMOCRACIA
}

\author{
marcial Murciano \\ Catedrático de Periodismo. Universitat Autònoma de Barcelona
}

\section{Resumen}

Los procesos de concentración en el sector de los medios de comunicación son un fenómeno característico de la moderna industria cultural. Diversas transformaciones producidas en la última década han alterado el difícil equilibrio entre los poderes económicos, políticos y sociales y la libertad de acceso al sistema de medios de comunicación. El artículo analiza estas tendencias en los procesos de concentración producidos durante la última década, valorando los retos que suponen los últimos cambios tecnológicos y la generalización de los grandes grupos multimedia para la libertad de expresión y la democracia.

\section{Palabras clave}

Procesos de concentración, libertad de expresión, grupos multimedia, sistema de medios, pluralismo democrático, cambio tecnológico y sociedad de la información.

\section{Abstract}

Concentration processes in the media sector are a characteristic phenomenon of the modern culture industry. Diverse transformations of the last decade have upset the delicate balance between the economic, political and social powers, on the one hand, and freedom of access to the media system on the other. This article analyses these trends in the concentration processes during the last decade, weighing up the challenges for freedom of expression and democracy set by the latest technological changes and the widespread growth of major multimedia groups.

Key Words

Concentration processes, freedom of expression, multimedia groups, media system, democratic pluralism, technological change and the information society. 


\section{LOS MEDIOS ANTE UN NUEVO ESCENARIO DE TRANSFORMACIONES SOCIALES}

Plantear a principios de este nuevo siglo el tema recurrente de la concentración en el sector de los medios de comunicación requiere en primer lugar situar este fenómeno, característico de la industria cultural, en la dinámica de su proceso histórico, por una parte; así como en el contexto de la serie de cambios profundos que han afectado a las sociedades industriales en las últimas décadas. Además, es preciso aportar una reflexión general sobre los mecanismos que pueden ayudar a evitar las tendencias de dominio en el sector de los medios tanto en los nuevos escenarios globales como en los locales.

El período que va desde finales de la década de los ochenta hasta nuestros días ha supuesto para los medios de comunicación un período de importantes y rápidas transformaciones. Sin ánimo de ser exhaustivos y sin la pretensión de establecer ninguna clase de jerarquía, me gustaría enumerar brevemente varios de estos cambios que considero fundamentales para comprender el nuevo contexto por el que atraviesan los medios de comunicación.

En primer lugar la dinámica del cambio tecnológico. En efecto, después de un período relativamente largo y denso de innovaciones tecnológicas que ha afectado al sistema de medios de comunicación de manera continuada durante el pasado siglo-recordemos que los ordenadores arrancan de la década de los cuarenta y los satélites de los cincuenta-, durante la segunda mitad de los ochenta y sobre todo en los noventa se concretaron y se difundieron ampliamente en todas las sociedades las nuevas tecnologías de comunicación, configurándose de forma rápida y singular nuevos medios, nuevos mercados y nuevos usos sociales. Para resumir brevemente esta tendencia de cambio que deseo señalar, creo que el fenómeno Internet ilustra y resume con claridad la capacidad del vector de la tecnología para dinamizar y transformar el conjunto del sistema de los medios de comunicación. Paralelamente al desarrollo de esta nueva tecnología, que desarrolla los nuevos medios de comunicación, los nuevos usos y consumos sociales, también se desarrollaron importantes sectores industriales y nuevos mercados vinculados a la implantación y difusión del cable, del satélite y los ordenadores personales. No cabe duda que la consolidación de la telefonía móvil, la tecnología digital y las redes interactivas han profundizado una dilatada y rápida tendencia de cambios en los medios de comunicación, sus industrias y sus mercados, en particular con la ampliación de su esfera de actividad económica que ha pasado a ser definida con propiedad como el "hipersector de la comunicación" (Díaz Nosty, 1995).

En segundo lugar, existe una nueva percepción y un reconocimiento generalizado de que la información y la comunicación, sus procesos y sus mercados, conforman el núcleo vital en el que se sustenta la emergente sociedad de la información o sociedad informacional o del conocimiento (Masuda, 1984; Castells, 2001), que basa una parte de su crecimiento y su desarrollo en la ampliación y la integración de las industrias de este nuevo hipersector de la comunicación (Webster, 1995). A esta nueva esfera industrial y de actividad humana no solo se integran los medios de comunicación tradicionales y sus productos, característicos de la revolución industrial, sino también las nuevas infraestructuras de transmisión como las redes convergentes de tecnología digital y tra- 
dicional y los nuevos terminales de recepción, cada vez más ubicuos, inteligentes e integradores de amplios procesos de comunicación (Mac Laughlin, 1982).

Un tercer elemento de cambio en el sector de los medios que me gustaría destacar, y que también se ha producido durante la última década, ha sido la nueva oleada de crecimiento y expansión de las actividades de los grandes grupos de comunicación, así como el amplio reconocimiento internacional que han obtenido como modelos de innovación y de gestión en el campo de la información, la comunicación y el entretenimiento. Es esta una dinámica que se ha generalizado ampliamente por todas las sociedades, del Norte y del Sur, pues hoy ya no son solamente reconocidos como referentes los nombres o las "marcas" de los grandes grupos multinacionales norteamericanos o japoneses -como Time/Warner, Disney, Microsoft, Sony o Nintendo-, sino que grupos como Telefónica o Prisa -por señalar dos empresas españolas- han adquirido este rango y dimensión, característico y exclusivo de las antiguas compañías multinacionales. Se trata por tanto de un modelo que se ha generalizado internacionalmente, tanto en los países más desarrollados como en los que se encuentran todavía y de una forma general en vías de desarrollo. En este sentido, México, Venezuela, Brasil o Argentina han favorecido durante la última década la consolidación de grandes grupos de comunicación como Televisa, Cisneros, Globo o Clarín, con crecientes intereses multinacionales e internacionalmente reconocidos.

Un cuarto elemento, ampliamente analizado y debatido durante la última década, ha sido la generalización del liberalismo como forma dominante de la economía y la política internacional contemporánea. La pugna mantenida durante el pasado siglo entre "capitalismo" y "socialismo" se decantó inexorablemente durante la última década. El hundimiento del "modelo soviético" y la apertura de China a la economía internacional han favorecido el establecimiento global de una economía basada en el libre mercado. En este contexto, el papel tradicional ocupado por el Estado en el desarrollo de los nuevos medios de comunicación (como en el caso de la gestión y la dirección del servicio público de la radio y la televisión, en el apoyo al establecimiento de las cinematografías nacionales o en el impulso de la industria local de programas) ha sido cuestionado. Hoy el Estado ha dejado de ser el gran actor dinamizador del desarrollo de los nuevos medios y su papel en el ordenamiento y la reglamentación del hipersector de la comunicación es limitado y muy a menudo controvertido (Blumler, 1992).

Por último y como consecuencia de los factores mencionados con anterioridad, durante los últimos años hemos asistido a la generalización de dos importantes dinámicas. Una primera, que impulsa el fuerte crecimiento económico e industrial en el sector de los medios y que ha favorecido la configuración de los medios como un amplio hipersector, con una cuota notable de incidencia en el PIB nacional. Y, en segundo lugar y de forma paralela, se ha acentuado la intensificación de las inversiones empresariales y financieras de los medios de comunicación en su expansión internacional. Ambas contribuyen a favorecer las tendencias tradicionales de dominio y de concentración en el sistema de los medios, tanto vertical como horizontalmente, tanto en el nuevo escenario global como en el tradicional del Estado nacional o en el emergente sector económico de los medios locales. 


\section{CONCENTRACIÓN Y MEDIOS DE COMUNICACIÓN: UNA PERSPECTIVA HISTÓRICA}

Por otra parte, y como es conocido, el tema de la concentración en los medios no es un fenómeno reciente, sino una constante que ha acompañado el desarrollo y la expansión del sector de la comunicación a lo largo de todo el siglo XX, de forma generalizada y con una intensidad variable en determinados períodos (Murciano, 1992).

Aunque en el análisis social, político e incluso cotidiano a menudo tiende a olvidarlo, lo cierto es que los "modernos medios de comunicación" (la prensa, el cine, la radio, las revistas y la televisión) adoptaron, desde sus inicios, la forma de organización industrial -primero industrias semiartesanales o ligeras, según algunos autores (Morin, 1966)-. En consecuencia, estas organizaciones empresariales han venido dedicándose desde entonces a producir, comercializar y difundir mercancías, aunque, por supuesto, mercancías con unas peculiaridades especiales, pues aunque contenían básicamente información, opinión y entretenimiento, eran componentes esenciales de "la nueva cultura de masas" de cualquier país desarrollado y un instrumento básico para la vida democrática de toda sociedad evolucionada (Ferguson, 1990).

Creo que este último aspecto es ciertamente el problema de fondo en el debate sobre el funcionamiento de los medios de comunicación en la democracia. El hecho de que los medios hayan adoptado como forma preferente y eficiente de funcionamiento un modelo comercial, y que por lo tanto la comunicación y sus productos se rijan por las leyes y las tendencias generales que orientan el mercado, ha condicionado estructuralmente sus formas, su evolución y sus problemas. Es por este motivo central que los medios tradicionales (prensa, cine, radio y televisión), en su desarrollo a lo largo de los últimos cien años, han tendido a establecer una competencia que les ha conducido, por un lado, al monopolio (geográfico y en su sector de actividad), y que, por otro, estas mismas empresas cada vez más concentradas en uno o varios sectores de los medios adquieran el carácter de grandes grupos de comunicación o grupos multimedia, que se expanden fuera de su ámbito geográfico inicial, con una proyección hacia los mercados internacionales (Guillou, 1984; De Miguel, 1993).

Aunque la tendencia general de la concentración en los medios es que el mercado o los diferentes mercados de la comunicación se orienten al monopolio, esta situación es excepcional y poco frecuente, pues en la práctica solo se da en los mercados locales. La concentración favorece sobre todo situaciones de oligopolio; es decir, el establecimiento de un número reducido de grupos de comunicación -dos, tres o cuatro, a lo sumo- que detentan en torno a un $70 \%$ de la oferta en un sector, aunque en ocasiones pueda representar la totalidad (Sánchez Tabernero y Carvajal, 2002).

Esta tendencia consolidada y preocupante en diferentes mercados de la comunicación hacia el oligopolio excluyente y generalizado (véase al respecto la posición actual de Microsoft mundialmente y en otros momentos la de las agencias de prensa internacionales) es el resultado de la amplia aceptación de las lógicas industriales y mercantiles en el funcionamiento y desarrollo em- 
presarial del sistema de los medios de comunicación social. Al igual que ha ocurrido a lo largo del siglo con otras ramas de la industria como el automóvil, la química, la farmacia o la banca, el mercado se vuelve oligopólico a consecuencia de la dinámica empresarial interna (aumento continuado de los costes de producción, aumento por consiguiente de la producción para amortizar los costes y de la cuota de mercado, para finalmente expulsar o absorber a la competencia); y de la dinámica expansiva empresarial, que una vez consolidadas las posiciones frente a los competidores realiza una expansión lógica hacia otros sectores (nuevos medios) y otros espacios (desde el global hasta los locales) como forma de valorizar sus activos y establecer sinergias expansivas (Quirós, 1991; y Medias Pouvoirs, 1994).

Si tomamos por ejemplo la trayectoria seguida por la prensa escrita, los diarios que mejor han representado la libertad de expresión, los diarios modestos y casi artesanales creados por grupos reducidos de personas con una vocación política, artística o cultural y más tarde informativa, han ido desapareciendo a lo largo del pasado siglo. En su lugar, aparecieron y se consolidaron las modernas e independientes empresas de prensa, con una administración eficaz dedicada a captar publicidad y un amplio grupo de profesionales especializados en las diversas tareas de redactar información. El gran periodismo moderno y empresarial, característico de la primera mitad del siglo XX, sufrió muy pronto una ola de concentraciones de carácter horizontal, es decir, la absorción de unas empresas por otras, en una verdadera lucha por consolidarse en el mismo sector de actividad comunicativa: la prensa primero, después las revistas, más tarde la radio y, por último, la televisión (Bagdikian, 1986).

Esta concentración clásica y horizontal se encuentra en los orígenes de la creación de las grandes cadenas de prensa, como las establecidas por R. Herst y E. Scripps, en los Estados Unidos, y con la consolidación de los primeros grandes grupos de comunicación en el sector de los medios. La concentración horizontal persigue por tanto la integración de una misma clase de medios, de una misma rama o sector de los medios, presionada por los beneficios empresariales. Primero fue la prensa, después las cadenas de radio y las revistas, más tarde las editoriales y por último la televisión. Desde finales de la pasada década, la concentración se desplaza de las industrias de medios a la industria de redes (telecomunicaciones), y de la electrónica a los servicios (Bustamante, 2002).

Por otra parte, también mientras se producía este fenómeno de concentración horizontal, los primeros grandes grupos de prensa empezaron a integrar actividades asociadas a la producción de prensa, produciéndose un segundo fenómeno de concentración vertical. Para una cadena o grupo de prensa en expansión, afectada por el fenómeno de concentración horizontal descrito, resultaba imprescindible controlar los diferentes sectores de la producción, empezando por las materias primas (como el papel) y finalizando por los talleres o los servicios de distribución. La integración vertical facilita la reducción de los costes y permite establecer sinergias dentro del grupo; la seguridad en la provisión de materias primas y una distribución de los productos comunicativos preferente. En definitiva, asegura la consolidación de una empresa dentro de su mercado. Todos los grandes grupos -empezando por los de prensa- han tendido a controlar empresas auxiliares y com- 
plementarias como las de publicidad o las agencias de noticias, con el objetivo de fortalecer su posición primero y el liderazgo más tarde (Bagdikian, 1986).

\section{EL SURGIMIENTO DE LOS GRANDES CONGLOMERADOS DE MEDIOS}

Un tercer momento en el fenómeno de la concentración lo constituye el surgimiento de los conglomerados en el sector de los medios de comunicación. Se trata de un fenómeno característico del período de expansión económica que se produce después de finalizar la Segunda Guerra Mundial. Podemos establecer, en términos generales, la aparición durante esta época de los actuales grupos multimedia y de dos modelos o clases de conglomerados (Curran, 1981).

En primer lugar, los conglomerados de medios, más tarde denominados grupos multimedia, tan presentes y activos en la actualidad. Entre ellos reconocemos a las grandes compañías mundiales de la comunicación como el Grupo AOL/ Time/Warner, generado tras la fusión primera de un gran grupo de prensa como Time, ya muy diversificado en los años ochenta, y un grupo consolidado del sector del entretenimiento, Warner Communications. El Grupo Time/Warner se ha convertido durante la década de los noventa del siglo pasado en el primer grupo mundial de comunicación, con presencia y actividades prácticamente en todos los sectores de la comunicación. La fusión con America On Line, le proporcionó además una presencia entre los emergentes sectores de actividad digital. La compañía Disney, después de absorber la cadena de televisión $A B C$, es otro de los modelos representativo de los grandes grupos concentrados. En Europa lo son Vivendi y Bertelsmann; en España, Prisa y Vocentro; y, en América Latina, Televisa y Globo son también ejemplos de esta clase de grupos muy concentrados y con actividades multimedia.

Un segundo tipo de conglomerados son los de configuración mixta y que se originan por la adquisición de un grupo o de diversas compañías de comunicación por parte de una empresa líder en otra actividad industrial ajena al sector de la comunicación. El ejemplo internacional más representativo en los años ochenta lo constituyó la compra que realizó Coca-Cola, una compañía tradicional instalada en la alimentación, al adquirir un estudio de cine como la Columbia. Este fenómeno de diversificación característico entre las grandes compañías industriales ha sido seguido por grupos petroleros, constructoras, empresas de servicios, etc. (Nabisco, Reynolds, etc.). En Europa, por ejemplo, la empresa constructora francesa Bouygues, que participó en la primera privatización de un canal público de televisión, se convirtió tras la adjudicación en uno de los primeros grupos audiovisuales europeos. Un proceso similar al que siguió el industrial Berlusconi, otro de los grandes líderes del sector audiovisual europeo, al entrar en el negocio de la televisión y la edición, a partir de una plataforma empresarial muy diversificada y con gran potencial financiero.

En general, el control producido por los conglomerados mixtos tiene un carácter especulativo y de diversificación de las actividades de su empresa base, sobre todo cuando ésta opera en un mercado ya maduro y con pocas expectativas de expansión. A menudo estas operaciones tienen un carácter meramente financiero y después de cierto tiempo las compañías de comunicación son 
revendidas en los mercados de valores con importantes plusvalías. Este es un fenómeno muy generalizado en Europa, donde los grandes bancos y los grupos empresariales líderes han ayudado con este criterio a la configuración de los grandes grupos de comunicación actuales (véase al respecto el ejemplo de Telefónica y el papel de los grandes grupos bancarios en España) (Reig, 1998).

Por lo demás, la concentración a través de procesos de conglomeración ha sido una forma de desarrollo y configuración de los grandes grupos de comunicación a lo largo de las recientes décadas de los ochenta y de los noventa. Muchos de estos nuevos conglomerados se han especializado en el sector de los medios de comunicación atraídos por las posibilidades de crecimiento que ofrecía la configuración de un nuevo hipersector industrial con enormes posibilidades de expansión. Esta es la trayectoria del sector audiovisual a escala europea y global. Así, el recientemente vendido grupo Vivendi francés, nacido en origen con actividades muy diversificadas, se especializó en el sector de los servicios, con notorias inversiones en el sector de los medios audiovisuales, como Canal +, Havas y Pathè. Y también las empresas de Murdock, con su creciente interés en el dominio de la televisión convencional y la digital en Europa.

Si hasta aquí hemos presentado un panorama sintético de las formas más características del proceso histórico de la concentración en los medios de comunicación, es oportuno señalar que desde finales de los ochenta se han producido la serie de cambios que hemos señalado al principio de nuestra exposición: la emergencia social e industrial de la nueva tecnología, concretada por los nuevos medios y los nuevos servicios de comunicación (las nuevas redes, el cable y los satélites; la apertura de una nueva era industrial en las sociedades más avanzadas, inducida por la revolución digital; así como las posibilidades que ofrece la convergencia digital entre los medios para nuevos desarrollos y las posibilidades multimedia que ofrece Internet a través de la banda ancha) que sitúa el problema de la concentración en otro nivel y en nuevos escenarios notablemente más complejos (Bustamante, 2002; y Rojo, 2003).

\section{EL NUEVO ESCENARIO DE LA CONCENTRACIÓN}

Por un lado, los nuevos soportes que configuran las redes de cable y de satélite han ampliado extraordinariamente el campo de la comunicación de una manera muy significativa y han roto los equilibrios funcionales que existían entre los medios tradicionales (prensa, radio, cine y televisión). Tomemos como ejemplo el marco político-cultural e informativo resultante de la existencia de una única televisión centralizada y de servicio público, en general en manos del Estado en casi toda Europa y creada y ampliada entre 1950 y 1970. En este contexto, la irrupción del cable y la televisión comercial, tanto por vía herziana como por satélite, han hecho incrementar de forma espectacular el número de canales, el número de horas de emisión, de producción y el mercado de programas. Si a principios de los ochenta se podían ver en Europa algo más de cincuenta canales de televisión, a finales de los noventa habían pasado a más de cuatrocientos de ámbito nacional (106 públicos y 303 privados) y más de mil de ámbito local, según fuentes del Observatorio Europeo del Audiovisual. Hoy, probablemente hemos pasado de 200.000 horas de emisión, en el pe- 
ríodo mencionado, a 2.000.000 de horas y a cerca de 10.000 canales en toda Europa (en la Europa definida por el Consejo de Europa, con más treinta miembros) (OEA, 2002).

Por otra parte, la revolución digital en curso y la convergencia digital a la que ésta conduce está aboliendo las especialidades sectoriales en las industrias de la información y la comunicación. Mientras que la industria de los medios anterior a la década de los noventa estaba diferenciada por las redes y los soportes de distribución que establecía, hoy esta realidad ha cambiado. Las redes de distribución de los periódicos, que terminaban en el kiosco, con un producto en soporte papel; la red de distribución del libro, que terminaba en las librerías o las bibliotecas; la red de distribución de la radio, que a través de las ondas termina en los altavoces de nuestra radio; y en el mismo sentido, la fotografía, el cine, el disco o la televisión habían constituido redes paralelas a las anteriores, diferenciadas por los soportes empleados y por las formas de codificación (escrita, sonora, visual, etc.) La nueva industria que desarrolla la tecnología digital, unifica medios y soportes diferentes. La tecnología binaria simplifica y unifica sectores industriales hasta ahora diferentes (la fotografía, el cine, el vídeo y la televisión). La tecnología de grabación y reproducción de imágenes está ahora unificada. Lo mismo ocurre para el sonido y para el escrito. La innovación digital conduce por una parte a una concentración en la industria, antes diversificada por sectores o ramas de especialización, y, por otra, a una concentración en las formas de distribución, donde parece emerger una red de distribución digital integrada que terminará en el ordenador/pantalla móvil o fija, como está ocurriendo con la telefonía. De este fenómeno de concentración industrial me gustaría señalar que emergen dos nuevos tipos de actores que hasta ahora tenían un peso limitado en el sector de los medios, por dedicarse a la transmisión de comunicación punto a punto, como es el caso de las compañías de telecomunicaciones (Telefónica entre otras empresas de telecomunicaciones es un buen ejemplo de esta tendencia) y los fabricantes de aparatos para la producción y recepción de información y entretenimiento, considerados hasta ahora como industriales del "hardware" y del "software" digitales (como Sony y Microsoft).

Un tercer elemento que hay que considerar, por su complejidad y por la dinámica de concentración que se observa en este nuevo escenario que ha introducido el cambio digital ha sido el papel central que ocupa Internet, entendida como la primera red operativa de la nueva tecnología, donde es posible encontrar interactividad, información y entretenimiento multimedia (prensa, conversaciones, radio, televisión, correo, consumo, educación y toda clase de servicios). Los grandes portales de entrada a la red han sido adquiridos en poco tiempo a precios exclusivos que solo han podido ser satisfechos por los grandes grupos como Disney, $\mathrm{MCl}$, ATT o Telefónica, cuyo nuevo portal Terra le costó 600 millones de dólares, aunque pierde unos 120 millones anuales. Estos portales hacia las nuevas redes de comunicación concentran la atención preferente de millones de los nuevos usuarios.

\section{LOS DESAFÍOS DE LA CONCENTRACIÓN: PODER ECONÓMICO E INFLUENCIA SOCIAL}

En cualquier caso, en este nuevo escenario que hemos esbozado la llegada de la nueva tecnología desarrolla dinámicas de cambio que debemos valorar de forma ambivalente. Por una par- 
te, la ampliación del campo de la comunicación y la integración de nuevas industrias en él nos permite considerar que cualquier nuevo actor debe siempre ser bienvenido. Se terminó el monopolio de IBM en el campo de los ordenadores, y los más de quinientos canales europeos actuales permiten pensar en un mayor espacio de libertad que las anteriores 30 empresas estatales. Sin embargo, el monopolio de IBM ha cedido su lugar central al que hoy posee Microsoft. Y los quinientos canales de televisión europeos transmiten en un 60 ó $70 \%$ las mismas series de ficción, las mismas películas y similares programas de entretenimiento.

En tal caso, el problema actual de la concentración en los medios reside, desde una amplia perspectiva político-cultural, en la concentración de poder económico y en la influencia social que se genera por esta posición de dominio. Y también, en qué medida esta permanente concentración altera los valores socioculturales, empresariales y políticos que constituyen el fundamento tradicional de las sociedades democráticas.

Resumiré esta problemática en tres grandes bloques de temas, sobre los que será preciso profundizar en posteriores análisis.

1. Desde el punto de vista general de una sociedad democrática abierta y participativa, el fenómeno de la concentración supone una alteración importante en la forma de la distribución social del poder, así como de las condiciones en las que se forjó el pluralismo democrático, la libertad de expresión y el contraste de las ideas.

A menor número de medios independientes, y de la posibilidad de crearlos en la nueva situación geopolítica y tecnológica, disminuye la posibilidad real de contrastar hechos, ideas y opiniones y también de expresarlos cotidianamente, tanto para los profesionales o comunicadores como para los ciudadanos. Como se ha señalado tantas veces y desde hace tanto tiempo (Hocking, 1947), el debate social, la agenda de los grandes temas de interés general y las opiniones alternativas quedan reducidas y las decisiones fundamentales son tomadas por elites especializadas o técnicas, minoritarias, sin el necesario y oportuno debate general (la redistribución del trabajo, la vivienda, la inmigración, o la guerra, como se ha visto recientemente con la declaración unilateral angloamericana en la Guerra de Irak, basada en información política tergiversada y equívoca).

2. Desde un punto de vista empresarial y desde la perspectiva de la pluralidad de actores en el mercado, la consolidación de los grandes grupos de comunicación o conglomerados multimedia supone una limitación clara tanto para entrar a competir en los nuevos mercados - como en el caso de los portales de Internet que hemos comentado- como en el diseño de programaciones alternativas (en la televisión) o en el desarrollo del "software" (Microsoft). Si los grandes grupos emiten en concurrencia películas americanas o espectáculos deportivos, un programador cultural no tiene mercado ni público para desarrollar una programación alternativa. La concentración refuerza las tendencias a la banalización cultural y el comercialismo en los medios, y es excluyente para los medios que se encuentran fuera del poder oligopólico. 
3. Por otra parte, la concentración pone al ciudadano común en una situación periférica con respecto a derechos fundamentales como son los de la libertad de expresión, el acceso a la información, la diversidad de las fuentes de información y la diversidad cultural. La posibilidad individual o colectiva de ejercer estos derechos se vuelve cada vez más escasa en nuestras sociedades industrialmente avanzadas y ricas. Es preciso y urgente avanzar en políticas concretas de intervención democrática en todos estos escenarios, con el objetivo central de redistribuir este poder básico y fundamental que todavía descansa en el sistema de los medios de comunicación y en la información de actualidad.

4. Por último, en esta última fase de la concentración que abre la revolución digital, existe una tendencia preocupante por el poder decisivo y directo que adquieren los grandes grupos financieros (los bancos) y la nueva industria de la información (por ejemplo, las compañías de telecomunicaciones o las de nueva tecnología como Microsoft) pues emergen como los actores decisivos para reorganizar en su propio beneficio el nuevo escenario de la comunicación y el sector de los medios. En este sentido, el liderazgo de la telefonía móvil mundial, que va a costar a la compañía británica Vodafone desembolsar hasta 100.000 millones de dólares, con apoyo de la gran banca y de los políticos de diversas naciones, pone en cuestión cualquier otro tipo de alternativas financieras y tecnológicas. Este tema es preocupante, sobre todo en un momento tan decisivo como el que estamos atravesando, de un gran cambio acelerado y en una sociedad donde el trabajo industrial está siendo sustituido por el emergente en los servicios y en la comunicación. El papel de árbitro que siempre ha mantenido el Estado en la moderna democracia se reduce y el mercado, ordenado ahora por los nuevos líderes empresariales, no asegura ninguno de los principios redistributivos que la democracia contemporánea debe asegurar al ciudadano que ahora debe situarse en un plano local y mundial al mismo tiempo. Es, sin duda, el momento de redefinir una nueva política para la comunicación social en el horizonte de la naciente sociedad del conocimiento, global y democrática.

\section{REFERENCIAS BIBLIOGRÁFICAS}

- Bagdikian, B. (1986): El monopolio de los medios de difusión, México: FCE.

- Blumler, J. (1992): Televisión e interés público, Barcelona: Bosch.

- Bustamante, E. (2002): Hacia un nuevo sistema mundial de comunicación, Barcelona: Gedisa (dos volúmenes).

- Castells, M. (2001): La galaxia Gutemberg, Barcelona: Plaza y Janés.

- Curran, J. (1981): Sociedad y comunicación de masas, México: FCE.

- De Miguel, J.C. (1993): Los grupos multimedia, Barcelona: Bosch.

- Díaz Nosty, B. (1995): Comunicación social. Tendencias, Madrid: Fundesco.

- Ferguson, M.(1990): Public Communication. The New Imperatives, Londres: Sage. 
- Guillou, B. (1984) : Les estratégies multimédias, París: Documentation Française.

- Hocking, W. (1947): Freedom of the Press, Chicago: U. Chicago Press.

- Mac Laughlin, J. (1982) : "La cartographie de l'industrie de l'information", Bulletin de I'IDATE", (Montpellier), num. 8.

- Masuda, Y. (1984): La sociedad informatizada como sociedad postindustrial, Madrid: Tecnos.

- Medias Pouvoirs (1994) : "Opa sur les médias? Les industriels dans la communication", Media Pouvoirs (Paris), num. 36.

- Morin, E. (1966): El espíritu del tiempo, Madrid: Tecnos.

- Murciano, M. (1992): Estructura y dinámica de la comunicción internacional, Barcelona: Bosch.

- Observatorio Europeo del Audiovisual (2003): Anuario Estadístico, Estrasburgo: OEA.

- Quirós, F. (1991): Curso de estructura de la información, Madrid: Dossat.

- Reig, R. (1998): Medios de comunicación y poder en España, Barcelona: Paidos.

- Rojo, P.A. (2003): Tecnología y contextos mediáticos, Sevilla: Comunicación Social.

- Sánchez Tabernero, A. y Carvajal, M. (2002): Media Concentration in the European Market, Pamplona: Eunsa. 